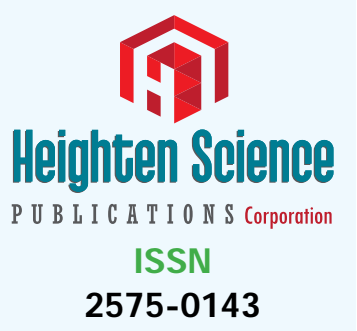

*Address for Correspondence: Scott Lilly, MD $410 \mathrm{~W} 10^{\text {th }}$ Ave 1 Maloney Building Columbus, OH 43210, Tel: (614) 293-7677; Fax: (614) 2935614; E-mail: Scott.Lilly@osumc.edu

Submitted: 04 March 2017

Approved: 17 May 2017

Published: 19 May 2017

Copyright: @ 2017 Makki N, et al. This is an open access article distributed under the Creative Commons Attribution License, which permits unrestricted use, distribution, and reproduction in any medium, provided the original work is properly cited.

Keywords: Acute myocardial infarction; Mortality; Weekend admissions; Weekday admissions
Research Article

\section{Procedure utilization, latency and mortality: Weekend versus Weekday admission for Myocardial Infarction}

\author{
Nader Makki ${ }^{3}$, David M Kline ${ }^{3}$, Arun Kanmanthareddy ${ }^{1}$, Hansie \\ Mathelier ${ }^{2}$, Satya Shreenivas ${ }^{2}$ and Scott M Lilly²* \\ ${ }^{1}$ Creighton University School of Medicine, Omaha NE, USA \\ 2Department of Cardiovascular Medicine, Ohio State's Wexner Medical Center, Columbus, Ohio, \\ USA \\ ${ }^{3}$ Center for Biostatistics, Department of Biomedical Informatics, the Ohio State University, \\ Columbus, Ohio, USA
}

\section{ABSTRACT}

Background: Due to variations in hospital protocols and personnel availability, individuals with myocardial infarction admitted on the weekend may be less likely to receive invasive procedures, or may receive them with a greater latency than those admitted during the week. Whether or not this occurs, and translates into a difference in outcomes is not established.

Methods: Using the Nationwide Inpatient Sample (2008-2011) database, we identified all patients admitted with a principle diagnosis of acute myocardial infarction. They were stratified by weekend or weekday admission. Baseline clinical characteristics, procedure utilization and latency to procedure were compared, and logistic regression models were constructed to assess the relationship between these variables and in-hospital mortality.

Results: Patient demographics and provider-related characteristics (hospital type, geography) were similar between weekend and weekday admission for myocardial infarction. Adjusted for covariates, we found that the odds of mortality for a weekend admission are $5 \%$ greater than for a weekday admission (OR: $1.05 ; 95 \% \mathrm{Cl}: 1.01$, $1.09, p=0.009$ ). For the utilization of an invasive procedure, we found that the odds of receiving a procedure for a weekend admission were $12 \%$ less than the odds for a weekday admission, adjusted for the other covariates (OR: $0.88 ; 95 \% \mathrm{Cl}: 0.86,0.91, \mathrm{p} \varangle 0.001$ ). In addition, we found that the time to procedure was an average of 0.18 days ( 4.32 hours) longer for weekend admissions compared to weekday admissions $(95 \% \mathrm{Cl}: 0.16,0.20$, $\mathrm{p} \varangle .001)$. However, we did not observe a significant difference in the overall length of stay for weekend and weekday admissions ( 0.004 days; $95 \% \mathrm{Cl}:-0.04,0.05, \mathrm{p}=0.87$ ).

Conclusion: In a large and diverse subset of patients admitted with myocardial infarction, weekend admission was associated with fewer procedures, increased latency to those procedures, and a non-significant trend towards greater in adjusted in-hospital mortality.

\section{INTRODUCTION}

Timely invasive angiography is associated with reductions in mortality in acute coronary syndromes (ACS) and is the current standard of care enforced by multiple guidelines [1,2]. While urgent and emergent angiography are routinely available at percutaneous coronary intervention (PCI)-capable hospitals; the latency to these procedures may be affected by availability of staff. Additionally, with Non-ST elevation acute coronary syndromes, guidelines suggest stable patients may receive angiography within 24-48 hours [3-5]. Whether or not staffing patterns dictate this interval, and what effect that may have on patient outcomes, is unclear. There have been a few 
reports that compare mortality rates among MI admitted on weekends compared to those admitted on weekdays, however, the findings have been inconsistent [6-10]. This has, in part, been due to difference in statistical adjustment, population size and proportion of PCI capable hospitals. No study to date has looked at the national practice patterns of the care of acute coronary syndromes presenting on weekends versus weekdays.

\section{METHODS}

\section{Data sources}

We utilized the National (Nationwide) In-patient Sample (NIS) database for this study. NIS is part of a family of databases and software tools developed for the Healthcare Cost and Utilization Project (HCUP) [11]. It is the largest publicly available all-payer inpatient health care database in the United States, yielding national estimates of hospital inpatient stays. Unweighted, it contains data from more than 7 million non-federal hospitals stay each year and when weighted it estimates more than 35 million hospitalizations nationally [11]. Given the de-identified nature of the NIS database, the Ohio State Data and Specimen Policy and Human Subjects Research policy did not require Institutional Review Board review for this study. We queried the 2008-2011 NIS database to identify all patients admitted with a principle diagnosis of acute myocardial infarction-AMI (code 410 of the International Classification of Diseases, 9th Revision, Clinical Modification). Furthermore, NIS contains demographic, clinical, and co-morbidity data on these patients in addition to including records of hospitalizations involving invasive cardiac procedures-cardiac catheterization, PCI, or coronary artery bypass grafting (CABG) [11].

\section{PATIENTS AND OUTCOMES}

Patient demographics (age, race, gender, and co-morbidities) and hospital characteristics (location, region, size, and teaching status) were collected. Individuals younger than 18 years of age and patients who sustained AMI during an admission for another diagnosis or procedure were excluded. We included patients who were admitted to hospitals with the principal diagnosis of AMI and were included in the NIS database between 2008 and 2011. The outcomes of interest were in-hospital mortality and health care utilization including length of stay, latency to procedure and procedure utilization for weekend versus weekday admission.

\section{Study variables}

The primary independent variable was admission on weekends (Saturday or Sunday) versus weekdays. Covariates included patient age on admission, gender, race, location and type of hospital (academic versus community). We calculated the Charlson Comorbidity index to quantify patient severity and comorbidities at admission [12,13]. The primary outcome variable was in-hospital mortality. We assessed length of stay, use of invasive procedures including PCI and CABG during hospitalization, the time from admission to procedure (in days), and overall incidence of cardiac arrest and cardiorespiratory shock during their stay.

\section{Statistical analysis}

All statistical analyses were conducted using the sampling weights provided in the data set to generate nationally representative estimates and appropriate standard errors. Summary statistics were calculated by year to describe the patient population under study. Logistic regression was used to estimate the effect of weekend admission on mortality and the utilization of invasive cardiac procedures. Linear regression models were used to assess the difference in length of stay and time to procedure between weekend and weekday admission. For each model, we fit unadjusted and 
adjusted models. Adjusted models included patient demographic covariates of age, gender, race, primary expected payer, year, median zip code income quartile, region, and month of admission. Adjusted models also included Charlson Score, an indicator of shock, and an indicator of cardiac arrest as markers of patient severity. A term was also included to adjust for the teaching status of the hospital that provided care. Statistical analysis was conducted using SAS 9.3.

\section{RESULTS}

Patient demographics and provider-related characteristics were similar between weekend and weekday admission for AMI. The majority of patients were male (60\%) and the average age was 67 years old. Table 1 depicts patient characteristics at admission for each year included in the study.

Unadjusted and adjusted model estimates of the effect of weekend admission are presented in table 2. Full model details are presented in the supplemental material. Adjusted for the other covariates in the model, we found that the odds of mortality for

\begin{tabular}{|c|c|c|c|c|c|c|c|c|}
\hline \multirow[t]{2}{*}{ Characteristic } & \multicolumn{2}{|c|}{2008} & \multicolumn{2}{|c|}{2009} & \multicolumn{2}{|c|}{2010} & \multicolumn{2}{|c|}{2011} \\
\hline & Weekend & Weekday & Weekend & Weekday & Weekend & Weekday & Weekend & Weekday \\
\hline Age (yrs.) & 65.3 & 67.9 & 67.5 & 67.4 & 67.3 & 67.5 & 67.2 & 67.7 \\
\hline Male (\%) & 60 & 60 & 61 & 61 & 60 & 60 & 61 & 61 \\
\hline White race (\%) & 78 & 78 & 77 & 76 & 75 & 76 & 75 & 75 \\
\hline $\begin{array}{l}\text { Charlson score for co- } \\
\text { morbidities }\end{array}$ & 5.41 & 5.46 & 5.49 & 5.51 & 5.47 & 5.51 & 5.59 & 5.66 \\
\hline \multicolumn{9}{|c|}{ Co-existing hemodynamic instability } \\
\hline Cardiac arrest (\%) & 3 & 3 & 3 & 3 & 3 & 3 & 3 & 3 \\
\hline $\begin{array}{c}\text { Cardiac/pulmonary shock } \\
\text { (\%) }\end{array}$ & 5 & 5 & 5 & 5 & 5 & 5 & 5 & 5 \\
\hline \multicolumn{9}{|c|}{ Primary payer } \\
\hline Medicaid (\%) & 55 & 57 & 55 & 56 & 55 & 56 & 56 & 58 \\
\hline Medicare (\%) & 5 & 5 & 6 & 6 & 6 & 7 & 6 & 6 \\
\hline Private Insurance (\%) & 30 & 29 & 28 & 28 & 29 & 27 & 27 & 26 \\
\hline Self-pay (\%) & 6 & 6 & 6 & 6 & 7 & 6 & 7 & 6 \\
\hline No charge (\%) & 1 & 1 & 1 & 1 & 1 & 1 & 1 & 1 \\
\hline \multicolumn{9}{|c|}{ Location/type of hospital } \\
\hline Rural (\%) & 12 & 12 & 11 & 10 & 12 & 12 & 10 & 10 \\
\hline Urban teaching (\%) & 46 & 44 & 45 & 43 & 44 & 43 & 44 & 43 \\
\hline Urban non-teaching (\%) & 43 & 44 & 45 & 47 & 44 & 45 & 46 & 48 \\
\hline \multicolumn{9}{|c|}{ Region } \\
\hline Northeast (\%) & 17 & 18 & 19 & 20 & 19 & 20 & 18 & 19 \\
\hline Midwest (\%) & 25 & 24 & 23 & 22 & 25 & 35 & 23 & 22 \\
\hline South (\%) & 40 & 41 & 42 & 41 & 38 & 37 & 40 & 40 \\
\hline West (\%) & 17 & 17 & 16 & 17 & 18 & 18 & 19 & 19 \\
\hline \multicolumn{9}{|c|}{ Median Zip code Income Quartile } \\
\hline $1(\%)$ & 28 & 28 & 29 & 28 & 29 & 29 & 29 & 29 \\
\hline 2 (\%) & 29 & 29 & 28 & 28 & 27 & 27 & 26 & 26 \\
\hline $3(\%)$ & 23 & 23 & 24 & 24 & 24 & 24 & 25 & 25 \\
\hline $4(\%)$ & 30 & 20 & 19 & 20 & 20 & 20 & 20 & 20 \\
\hline
\end{tabular}

*The Charlson Comorbidity Index is a method of categorizing comorbidities of patients based on ICD diagnosis codes found in administrative data. Each comorbidity category has an associated weight (from 1 to 6), based on the adjusted risk of mortality or resource use, and the sum of all the weights results in a single comorbidity score for a patient

Table 2: Estimates for comparing patients admitted on the weekend to those admitted on weekdays.

\begin{tabular}{|c|c|c|c|c|c|c|}
\hline & \multicolumn{3}{|c}{ Unadjusted Estimates } & \multicolumn{3}{c|}{ Adjusted Estimates } \\
\hline Outcome & OR & $\mathbf{9 5 \%} \mathbf{C l}$ & $\mathbf{p}$-value & $\mathbf{O R}$ & $\mathbf{9 5 \%} \mathbf{C l}$ & $\mathbf{p}$-value \\
\hline Mortality & 1.06 & $(1.03,1.09)$ & $\varangle .001$ & 1.05 & $(1.01,1.09)$ & 0.0089 \\
\hline Utilization of procedure & 0.92 & $(0.90,0.94)$ & $\varangle .001$ & 0.88 & $(0.86,0.91)$ & $\varangle .001$ \\
\hline Outcome & Difference & $\mathbf{9 5 \%} \mathbf{C l}$ & $\mathbf{p}$-value & Difference & $\mathbf{9 5 \%} \mathbf{C l}$ & $\mathbf{p}$-value \\
\hline Time to procedure (days) & 0.15 & $(0.13,0.17)$ & $\varangle .001$ & 0.18 & $(0.16,0.20)$ & $\varangle .001$ \\
\hline Length of Stay (days) & -0.01 & $(-0.05,0.03)$ & 0.5984 & 0.004 & $(-0.04,0.05)$ & 0.8663 \\
\hline
\end{tabular}


a weekend admission are 5\% greater than for a weekday admission (OR: 1.05; 95\% CI: 1.01, 1.09). For the utilization of an invasive procedure, we found that the odds of receiving a procedure for a weekend admission were $12 \%$ less than the odds for a weekday admission, adjusted for the other covariates (OR: 0.88; 95\% CI: 0.86, 0.91). Not only were the odds of receiving a procedure reduced for a weekend admission, but we also found that the time from admission to the administration of the procedure was also increased for weekend admissions. We found that the time to procedure was an average of 0.18 days ( 4.32 hours) longer for weekend admissions compared to weekday admissions ( $95 \%$ CI: $0.16,0.20)$. However, we did not observe a significant difference in the overall length of stay for weekend and weekday admissions $(0.004$ days; $95 \%$ CI: $-0.04,0.05)$.

\section{DISCUSSION}

The present study, analyzing a large cohort that is representative of all US inpatient stays over 4 years, has demonstrated the following: 1) Weekend admission for myocardial infarction was associated with fewer invasive cardiac procedures; 2) an increased latency to those procedures; and; 3 ) increased in-hospital mortality.

Previous studies have alluded to a "Weekend effect" in which lower staff and resource availability was related to increases in mortality for life-threatening conditions [8,14]. In various studies this finding has been linked to the acuity of the presenting illness [15], teaching status of the hospital [14], or the timely utilization of potentially life-saving procedures [14]. Using a statewide database, Kostis et al. observed higher 30-d and 1 year mortality among patients with myocardial infarction admitted on weekends that became non-significant only after adjusting for lower utilization of invasive cardiac procedures [16]. In a large cohort, our study showed that there was less use of invasive procedures, longer latency to those procedures, and a trend towards increased mortality in patients admitted on weekends with AMI. However, there are important differences to consider between our study and what has been published in literature: 1) we adopted different statistical adjustment models specifically pertaining to the Charlson score; 2 ) our population sample was larger; and 3) we assessed in-hospital mortality only unlike Kostis et al. who examined 30 day and 1 year mortality. In addition to difference in study structure, other possible explanations for differences in results compared to previous studies include: 1) improved level/quality of hospital staffing over recent years; and 2) proliferation of PCI -capable hospitals since publication of the Kostis article, and a greater proportion of patients in our study being admitted to PCI capable hospitals ( $\sim 67 \%)$ compared to the Kostis study (25\%). This may account for some of the observed difference.

Others have identified no difference in mortality rates between weekend and weekday admissions for acute myocardial infarction. Bell et al. reported no difference in mortality (OR 1.03 95\%CI 1.00-1.06) among 160,220 myocardial infarction patients stratified by weekend versus weekday admission over a 10 -year period [8,17]. Similarly, it was demonstrated that patients admitted with ST-elevation myocardial infarction during the weekends have similar short and long-term survival rates as patients admitted during weekdays over a period of 15 years (1985-2000) [18]. However, in these reports-mostly based out of Japan/Europe-the similar mortality between weekend and weekday admissions was attributed to proper availability of staff and the ability to perform invasive procedures readily during weekends. It is likely that given systems in place for STEMI reduce practice variation, and the differences we observe herein reflect primarily the management of UA/NSTEMI, where care may be more individualized.

Going forward it is important to note that the "weekend effect" varies depending on type of ACS- specifically whether it is STEMI or not. In current day practice, management 
of STEMI is governed by benchmarks for door to balloon time. Therefore, weekend admissions with lower staffing resources and potential delay in door to balloon time are associated with increased mortality in patients with STEMI [19]. However, for UA/ NSTEMI we are allowed to individualize depending on presentation and associated risk features. It is probably this population that drives all the outcomes and partly responsible for the discordance in results among the different published studies so far. The "weekend effect" may subsequently depend on type of ACS with a recent study demonstrating that the increased mortality associated with weekend admissions is more notable in NSTEMI as compared to STEMI [15]. In a recent analysis of the NIS database, Agrawal et al. demonstrated that weekend admissions for Non ST-elevation myocardial infarctions are associated with lower rate of coronary angiography and higher In-hospital mortality. However, they noted that mortality became similar after adjusting for rate of utilization of an early invasive strategy (OR 1.01; 95\% CI 0.99 to 1.03; $p=0.11$ ) [20]. In comparison, we demonstrated in our analysis that mortality was similar between weekend and weekday admissions after adjusting for co-morbidities (specifically cardiogenic shock and cardiac arrest) irrespective of lower rate of utilization of invasive strategy on weekends.

Our study has important strengths. We were able to capture a large sample of patients utilizing the NIS database and performed extensive statistical adjustment to account for effect of confounding variables. Furthermore, our study highlights an important message, which is that weekend practices for patients admitted with AMI are associated with increased mortality, reduced utilization of invasive procedures and increased latency to procedures. However, our study has a number of limitations that merit consideration. First, our study was conducted between 2008 and 2012, which is a bit old. This may affect results especially given the wide availability of highly sensitive troponin assays in recent years. Second, we were limited by the ability to analyze only in-hospital mortality, and there may be differences in intermediate- and longer-term outcomes. In addition, we were not able to perform separate analysis on STEMI versus UA/NSTEMI, which could have a significant bearing when it comes to the "weekend effect". Another limitation was lack of data on patients' clinical status such as congestive heart failure, arrhythmias, and recurrent angina. This paper and others like it provide more a rationale to proceed with a prospective multi-institution study examining the role of delayed invasive procedures for AMI, especially UA/NSTEMI.

\section{CONCLUSION}

In a large and diverse set of patients with MI, weekend admission was associated with fewer procedures, increased latency to those procedures, and increased inhospital mortality. This finding suggests that the practice patterns associated with reduced staffing and resource availability might result in poorer in-hospital patient outcomes.

\section{REFERENCES}

1. De Luca G, Suryapranata H, Ottervanger J P, Antman EM. Time delay to treatment and mortality in primary angioplasty for acute myocardial infarction: every minute of delay counts. Circulation. 2004; 109: 1223-1225. Ref.: https://goo.gl/4yXYAC

2. Amsterdam EA, Wenger NK, Brindis RG, Casey DE J r, Ganiats TG, et al. 2014 AHA/ACC Guideline for the Management of Patients with Non-ST-Elevation Acute Coronary Syndromes: a report of the American College of Cardiology/American Heart Association Task Force on Practice Guidelines. J Am Coll Cardiol. 2014; 64: 139-228. Ref.: https://goo.gl/p7MvNR

3. Fox KA, Clayton TC, Damman P, Pocock SJ , de Winter RJ , et al. Long-term outcome of a routine versus selective invasive strategy in patients with non-ST-segment elevation acute coronary syndrome a meta-analysis of individual patient data. J Am Coll Cardiol. 2010; 55: 2435-2445. Ref.: https://goo.gl/mj7GyY 
4. Alfredsson J , Clayton T, Damman P, Fox KA, Fredriksson M, et al. Impact of an invasive strategy on 5 years outcome in men and women with non-ST-segment elevation acute coronary syndromes. Am Heart J . 2014; 168: 522-529. Ref.: https://goo.gl/GzWruD

5. Lagerqvist B, Husted S, Kontny F, Stahle E, Swahn E, et al. 5-year outcomes in the FRISC-II randomised trial of an invasive versus a non-invasive strategy in non-ST-elevation acute coronary syndrome: a follow-up study. Lancet. 2006; 368: 998-1004. Ref.: https://goo.gl/w7iA9z

6. I neid $\mathrm{H}$, Fonarow GC, Cannon $\mathrm{CP}$, Palacios IF, Kilic $\mathrm{T}$, et al. Impact of time of presentation on the care and outcomes of acute myocardial infarction. Circulation. 2008; 117: 2502-2509. Ref.: https://goo.gl/2GcEwB

7. Hong J S, Kang HC, Lee SH. Comparison of case fatality rates for acute myocardial infarction in weekday vs weekend admissions in South Korea. Circ j. 2010; 74: 496-502. Ref.: https://goo.gl/GsAuhv

8. Bell CM, Redelmeier DA. Mortality among patients admitted to hospitals on weekends as compared with weekdays. N Engl J Med. 2001; 345: 663-668. Ref.: https://goo.gl/10f16l

9. Matsui K, Kojima S, Sakamoto T, Ishihara M, Kimura K, et al. Weekend onset of acute myocardial infarction does not have a negative impact on outcome in J apan. Cir J . 2007; 71: 1841-1844. Ref.: https://goo.gl/TDrEG0

10. Turin TC, Kita $Y$, Rumana N, Sugihara H, Morita $Y$, et al. Incidence, admission and case-fatality of acute myocardial infarction: weekend versus weekday in a J apanese population: 16-year results from Takashima AMI Registry (1988-2003). Eur J Epidemiol. 2009; 24: 93-100. Ref.: https://goo.gl/kgzf8C

11. Bekkers S, Bot AG, Makarawung D, Neuhaus V, Ring D. The National Hospital Discharge Survey and Nationwide Inpatient Sample: the databases used affect results in THA research. Clin Orthop Relat Res. 2014; 472: 3441-3449. Ref.: https://goo.gl/TCkG9h

12. Charlson ME, Pompei $P$, Ales KL, MacKenzie CR. A new method of classifying prognostic comorbidity in longitudinal studies: development and validation. J Chronic Dis. 1987; 40: 373-383. Ref.: https://goo.gl/VKbL4k

13. D'Hoore W, Sicotte C, Tilquin C. Risk adjustment in outcome assessment: the Charlson comorbidity index. Methods Inf Med. 1993; 32: 382-387. Ref.: https://goo.gl/ZmCwwo

14. Cram P, Hillis SL, Barnett M, Rosenthal GE. Effects of weekend admission and hospital teaching status on in-hospital mortality. Am J Med. 2004; 117: 151-157. Ref.: https://goo.gl/w2qlkR

15. Khoshchehreh M, Groves EM, Tehrani D, Amin A, Patel PM, et al. Changes in mortality on weekend versus weekday admissions for Acute Coronary Syndrome in the United States over the past decade. Int J Cardiol. 2016; 210: 164-172. Ref.: https://goo.gl/B5NvCL

16. Kostis WJ, Demissie K, Marcella SW, Shao YH, Wilson AC, et al. Weekend versus weekday admission and mortality from myocardial infarction. N Engl J Med. 2007; 356: 1099-1109. Ref.: https://goo.gl/SOgnON

17. Bell CM, Redelmeier DA. Waiting for urgent procedures on the weekend among emergently hospitalized patients. Am J Med. 2004; 117: 175-181. Ref.: https://goo.gl/a1YzAi

18. Snelder SM, Nauta ST, Akkerhuis KM, Deckers J W, van Domburg RT. Weekend versus weekday mortality in ST-segment elevation acute myocardial infarction patients between 1985 and 2008. Int J Cardiol. 2013; 168: 1576-1577. Ref.: https://goo.gl/PvobXa

19. Magid DJ , Wang Y, Herrin J , MCNamara RL, Bradley EH, et al. Relationship between time of day, day of week, timeliness of reperfusion, and in-hospital mortality for patients with acute ST-segment elevation myocardial infarction. J AMA. 2005; 294: 803-812. Ref.: https://goo.gl/lli7Hz

20. Agrawal S, Garg L, Sharma A, Mohananey D, Bhatia N, et al. Comparison of Inhospital Mortality and Frequency of Coronary Angiography on Weekend Versus Weekday Admissions in Patients With Non-ST-Segment Elevation Acute Myocardial Infarction. Am J Cardiol. 2016; 118: 632-634. Ref.: https://goo.gl/lJ wc6A 